\title{
Clinical Handover among Physicians: A Survey in a North Eastern Italian Academic Hospital
}

\author{
Rosanna Quattrin (Corresponding author) \\ Azienda Sanitaria Universitaria Integrata di Udine \\ 15 P.le Santa Maria della Misericordia, Udine 33100, Italy \\ Tel: +39 432559903 E-mail: rosanna.quattrin@asuiud.sanita.fvg.it \\ Giulio Menegazzi \\ Department of Medical and Biological Sciences \\ 50 Via Colugna, Udine 33100, Italy \\ Tel: +39 $432559161 \quad$ E-mail: giulio.menegazzi@uniud.it

\section{Adriana Moccia} \\ Azienda Sanitaria Universitaria Integrata di Udine \\ 15 P.le Santa Maria della Misericordia, Udine 33100, Italy \\ Tel: +39432554487_E-mail: adriana.moccia@asuiud.sanita.fvg.it
}

\section{Carla Londero}

Azienda Sanitaria Universitaria Integrata di Udine

15 P.le Santa Maria della Misericordia, Udine 33100, Italy

Tel: +39432559208Ｅ-mail: carla.londero@asuiud.sanita.fvg.it

Silvio Brusaferro

Department of Medical and Biological Sciences

50 Via Colugna, Udine 33100, Italy

Tel: +39432559206_E-mail: silvio.brusaferro@uniud.it

Received: March 31, 2016 Accepted: May 23, $2016 \quad$ Published: March 7, 2017

doi:10.5296/jss.v3i1.9242 URL: http://dx.doi.org/10.5296/jss.v3i1.9242 


\section{Abstract}

Transferring essential information and responsibility for patient care from one health care provider to another is an integral component of patient safety in a hospital. This study aims to collect physician interests and needs about handover before introduction of a standardized tool in a North Eastern Italian Academic Hospital (AH). From October 2014 to December 2014 all physicians working in the $\mathrm{AH}$ were asked to fill a web-based questionnaire concerning currently adopted methods to clinical handover and their perception on its. Response rate was 10.5\% (90/853). 63.3\% (57/90) of physicians showed maximal agreement with the statement "communication failures are related to adverse events" and 40\% (36/90) of them completely agreed that "improving handover makes work safer". Among actually used handover methods (total answers: 157), verbal communication was referred $45.2 \%$ times, written notes $26.1 \%$, electronic supports $21.7 \%$. The most frequently reported obstacles to an effective handover (107 answers) were interruptions (27.1\%), absence of hospital written protocols $(25.2 \%)$ and colleague's inability to provide further information when required (17.7\%). Respondents interested to test a new handover procedure were $48.9 \%$ (44/90). The low response rate and the fact that only half of respondents were interested in experimenting a new procedure stress the little relevance given by physicians to handover as a key process for patient safety. Furthermore, hazardous methods of transferring information such as verbal communication and non-structured text, combined with referred handover obstacles, suggest the necessity of developing a hospital policy for clinical handover among physicians.

Keywords: Clinical handover, Physicians, Survey

\section{Introduction}

In hospital communication is very important and also the way in which information is conveyed plays its role, especially if we consider that this aspect is often left behind in the hierarchy of values of the healthcare workers. There are many studies on communication in the health care setting. Professional communication practices or strategies and team collaboration have a relationship with improved patient outcomes and patient safety and there is strong evidence that improving communication also increases staff satisfaction (Clark \& Greenawald, 2013; Siedlecki \& Hixson, 2015).

To transfer essential information and responsibility for patient care from one health care provider to another (a practice commonly referred as handover) is an integral component of communication in healthcare setting (ACSQHC, 2005; Ong \& Coiera, 2011; Mardis et al., 2015). Clinical handover is the transfer of professional responsibility and accountability for some or all aspects of care for a patient, or group of patients, to another person or professional group on a temporary or permanent basis (ACSQHC, 2009; ACSQHC, 2011).

An efficacious handover supports transition of critical information and continuity of care and treatment. Clinical environments are dynamic and complex, presenting many challenges for effective communication. The literature highlights the effects of ineffective handover such as wrong patients identification, wrong site surgery and, sometimes, patient death (Rabøl et al., 
2011; Thomas et al., 2013). The communication methods impact on the effectiveness and efficiency of handover as well as patient safety.

In 2006 Joint Commission International (JCI) introduced a national patient safety goal to develop procedures for the handover of patients among clinicians. The input came from the Sentinel Event Advisory Group based on data collected in accredited hospitals which identified goals with the potential to protect patient safety (JCI, 2014).

This study aims to collect physician interests and needs regards to handover before introduction of a hospital standardised tool in an Academic Hospital (AH).

\section{Methods}

The survey was carried out from October 2014 to December 2014. It consisted in administering to all physicians, working in a North Eastern Italian $\mathrm{AH}$, an anonymous web-based questionnaire concerning currently adopted methods to transition of care and their perception on handover issues.

The questionnaire (available on request) was developed on the basis of a pertinent literature review about handover topic by focusing on published audit and root cause analysis (Rabøl et al., 2011) and models for building a standardised handover protocols used in other countries (Quattrin et al., 2014). It included 13 items regarding physician general characteristics (n.3), actually used patients information transition of care methods (n.4), obstacles to correct shift-to-shift handover (n.1), perception and interests on handover issue (n.5). Multiple answers were allowed when inquiring the methods and obstacles to handover, while perceptions and interests in the topic required a single answer or an expression of agreement to certain statements through a Likert scale from 1 to 5.

Data collected from the questionnaires were entered in an Excel spreadsheet and were analysed using the statistical software SPSS, version 20.

\section{Results}

Response rate was 10.5\% (90/853). Among these, 76.7\% (69/90) was physicians and 23.3\% (21/90) residents. In regard to occupational area, 52.2\% (47/90) of respondents worked in surgical operative units (OUs), 26.7\% (24/90) in services OUs and 21.1\% (19/90) in medical OUs.

Table 1 shows actually used handover methods and settings actually where they took place during the day. $24.4 \%$ (22/90) of physicians referred that they transfer patient information only verbally, $94 \%$ (85/90) of respondents reported that they often have the opportunity to share opinions face-to-face on elements considered important. 42.2\% (38/90) of physicians referred that in their units nursing staff shares handover with medical equip and vice versa, $36.7 \%(33 / 90)$.

Table 2 shows reasons about physicians' experienced obstacles to an effective handover. $22.2 \%(20 / 90)$ of respondents declared that "there are not obstacles and the handover is excellent”. 
Physicians were also asked to express their perception of relevance to the topic of risk management and effects on daily routine. The statement "a poor shift-to-shift handover may lead to clinical errors, even major ones" met maximal agreement ( 5 on the Likert scale) in $63.3 \%$ (57/90) of cases, while only 9 subjects (10\%) gave a score of 3 or less. The other statements however provided a more mixed distribution of agreement. "I think that an easier management of handover would make my work safer" reached a score of 5 in 40\% (36/90) of the responses, 4 in 25.6\% (23/90), 3 in 23.3\% (21/90). The frequency of total disagreement with this statement ( 1 on the Likert scale) was 6.7\% (6/90). The declaration "important patient information are lost during shift change" showed a maximal agreement in $12.2 \%$ $(11 / 90)$ cases, which is inferior to the frequency of minimal agreement [14.4\% (13/90)].

To the question "which instrument would be more useful (reliable) to simplify the handover among colleagues?", physicians reported "to promote opportunities for direct transfer of information" in 34.1\% (46/135) of answers [multiple choice], "electronic tools with pre-filled form" in 23.7\% (32/135), "word document with free entry" in 17.8\% (24/135), "checklist paper form with tick" in $11.1 \%$ (15/135), "not need any of these” in 4.4\% (6/135) and "other" in the remaining cases.

48.9\% (44/90) of respondents reported to be interested in testing for a period with their colleagues a different way of patient information transfer to evaluate its effectiveness. In surgical area physicians showed a higher percentage of engagement [63.2\% (12/19)]. 26.7\% (24/90) of all respondents did not interest in testing but they wanted to be more informed on handover issue and 22.2\% (20/90) referred that the current situation was optimal. In surgical area physicians showed a higher percentage of interest [63.2\% (12/19)].

Analysing the database of hospital incident reporting system, the adverse events/near misses number in the area of management information and in particularly regards to an ineffective handover among healthcare workers was 92 in 2014 equal to 12.5\% (92/736) of all reported and to 0.22 per 100 admissions.

\section{Discussion}

The low response rate and the fact that only half of respondents were interested in experimenting a new procedure stress the little relevance given by physicians to handover as a key process for patient safety. Furthermore, hazardous methods of transferring information such as verbal communication and non-structured text, combined with referred handover obstacles, and the percentage of incident reporting linked to an ineffective transmission of clinical information among healthcare personnel suggest the necessity of a hospital standardized approach to handover communication in the hospital.

The National Transition of Care Coalition defines "transitions of care" (handover) as "the movement of patients between health care location, providers or different levels of care within the same locations as their conditions and care needs change” (NTOCC, 2015).

The first comment regards to verbal communication used more frequently for the handover. It was a hazardous methods of transferring information. A verbal report has the potential weakness of poor retention of information by the receiver, and there may be discrepancies 
between the reported status and the actual patient status because of memory failure on the part of the reporter (Frankel, 2012). Literature shows that after five handover cycles, only $2.5 \%$ of patient information was retained using the verbal-only handover method while 85.5\% was retained when using the verbal with note taking method and 99\% was retained when a printed handout containing all patient information was used (Bhabra et al., 2007).

Standardised checklist was a rare method reported by the respondents even if a structured pre-printed form had already been shown in literature to be the most effective (ACSQHC, 2009; ACSQHC, 2011; Quattrin et al.; 2014). Checklists help in managing information without missing important patient data (Salzwedel et al., 2013), improve the quality of handover process and enhance quality of care and patient safety (WHO, 2007; Wong et al., 2008; Matic et al, 2011). A study (Pothier, 2005), examining three different methods of handoff, found that the one demonstrating the greatest amount of information retention involved utilisation of a pre-printed sheet containing patient information with verbal report (96\%-100\%), followed by note taking and verbal report method (31\%-58\%), and lastly, only verbal report (0-58\%). The insertion of incorrect information was observed in the verbal-only method.

In this study a high percentage of physicians reported that handover took place at shift changes making presuppose that, for the respondents, the primary purpose of the handover was to convey essential patient care information, to promote continuity of care, to meet therapeutic goals and to assure the safe transfer care of the patient to a colleague.

In line with the literature, physicians referred that their experienced obstacles to an effective handover were, in order of frequency, interruptions and distractions [20], lack of structured policies and procedures about handover (Malekzadeh et al., 2013; Quattrin et al., 2014) and colleague's inability to provide further information when required. Interruptions are accepted in healthcare setting even if several studies suggest that they origin human error (McGillis Hall, 2010; Brixey et al., 2010) because they can trigger cognitive failures such as lapses in attention, memory or perception. Therefore, during handover critical information could be lost and to complete or to initiate clinical tasks could failure (Wong et al., 2008; Westbrook et al., 2010).

A peculiar finding of the present research was that none of the respondents reported the incomplete and illegible information contributing to failed communication process. It is very strange because in the hospital there was not electronic medical record and the Authors supposed that the unclear handwriting in the clinical documents could be a problem. Interesting is the fact that a quarter of the sample declared that handover in his ward was excellent.

Answers regards to perceptions on handover show that most of the respondents are aware that a poor transferring of information among physicians may lead to serious clinical errors and an easier management of handover would make their work safer. This knowledge could be a "picklock" to make participative the physicians in the standardization of the handover process because they believe that the primary purpose of the shift handover is to provide a safe and high-quality care (Wong et al., 2008). 
About instruments more useful to simplify the handover, the respondents reported as the first the necessity to promote opportunities for direct transfer of information. This answer reveals the need of social interaction to debrief, clarify information and update knowledge. Literature reports that face to face communication is the best means for ensuring responsibility for patient care and it assists handover to be an interactive process, where opportunity for questioning and verifying is enabled between the giver and receiver of the information (ACSQHC, 2009). Some studies found that face-to-face handoffs were preferable to those that exclude a face-to-face conversation (Arora et al., 2008; Friesen et al., 2008). In fact many other modalities, besides the written ones, are involved in the handover communication such as paralinguistic features (pitch, pace, intonation, hesitations) and non verbal behaviour including information delivered throughout gesture, posture, bodily orientation, facial expression, eye contact and physical distance (Frankel et al., 2012).

In this study electronics tools were considered more useful than word document and checklist. Computerised tools should be designed to aid communication between doctors, during handover preparation, as well as the handover itself and subsequent activities. These electronic solutions are designed to improve the accuracy of information transfer, but do not take into consideration the behavioural aspects and, therefore, do not always assess all the functions of handover (Frankel et al., 2012).

Surgical area physicians demonstrated an interest to implement a new standardized methods for clinical handoff while a third of the sample did not show this interest but it wanted only to be more informed on handover issue.

In conclusion many suggestions derived by the results. First, the safety culture in regards to handover proved to be immature, therefore it is essential to spread the culture that a poor shift report, with essential information not documented properly, may contribute to an adverse outcome. Second, the heterogeneity of methods and tools used for handover in the hospital determines the priority to standardize the process of conveying essential patient information. Third, the approach to implement a standardized handover communication will be participative, not top-down, that is each handover will be designed to fit the workload and users will be included in the design process. Remarkably, the same tool may not be perfectly suitable for every discipline and organization, and every team can adjust it to its own needs (Quattrin et al., 2014).

\section{Conclusion}

This study represents a primary level of analysis of the context and early involvement of the handover users in a hospital. It gave significant recommendations to orienting the organization to the adoption of methods that increased the effectiveness of handover among healthcare workers at several patient's care levels.

\section{References}

Arora, V., \& Johnson, J. (2006). A model for building a standardized hand-off protocol. Joint Commission Journal on Quality and Patient Safety, 32(11), 646-655. 
Australian Commission on Safety and Quality in Health Care. (2009). A framework to support clinical communication. [Online]. Available: http://www.safetyandquality.gov.au/ implementation-toolkit-resource-portal/resources/simple-solution-development/SHARED\%2 0Resource\%20Guide.pdf (May 13, 2016)

Australian Commission on Safety and Quality in Health Care. (2009). The OSSIE guide to clinical handover improvement. Sydney, Australia: Australian Commission on Safety and Quality in Health Care (ACSQHC).

Australian Commission on Safety and Quality in Health Care. (2011). Implementation Toolkit for Clinical Handover Improvement. Sydney, Australia: Australian Commission on Safety and Quality in Health Care (ACSQHC).

Australian Council for Safety and Quality in Health Care. (2005). Clinical handover and patient safety literature review report. [Online]. Available: http://www.safetyandquality.gov. au/wp-content/uploads/2012/01/clinhovrlitrev.pdf (May 3, 2015)

Bhabra, G., MacKeith, S., Monteiro, P., \& Pothier, D. D. (2007). An experimental comparison of handover methods. Annals of the Royal College of Surgeons England, 89, 298-300. http://dx.doi.org/10.1308/003588407X168352

Brixey, J. J., Robinson, D. J., Turley, J. P., \& Zhang, J. (2010). The roles of MDs and RNs as initiators and recipients of interruptions in workflow. International Journal of Medical Informatics, 79(6), e109-e115. http://dx.doi.org/10.1016/j.ijmedinf.2008.08.007

Clark, R. C., \& Greenawald, M. (2013). Nurse-physician leadership: insights into interprofessional collaboration. The Journal of Nursing Administration, 43(12), 653-659. http://dx.doi.org/10.1097/NNA.0000000000000007

Frankel, R. M., Flanagan, M., Ebright, P., Bergman, A., O'Brien, C. M., Franks, Z., Allen, A., Harris, A., \& Saleem, J. J. (2012). Context, culture and (non-verbal) communication affect handover quality. BMJ Quality and Safety, 21(Suppl 1), i121-i128. http://dx.doi.org/10.1136/bmjqs-2012-001482

Friesen, M. A., White, S. V., \& Byers, J. F. (2008). Handoffs: Implications for Nurses. In R. G. Hughes (Ed.), Patient Safety and Quality: An Evidence-Based Handbook for Nurses (Vol. 2, pp. 285-332). Rockville, MD: Agency for Healthcare Research and Quality.

Joint Commission International. (2014). Joint Commission International Standards for Hospitals (5th ed.). USA: Oakbrook Terrace.

Malekzadeh, J., Mazluom, S. R., Etezadi, T., \& Tasseri, A. (2013). A standardized shift handover protocol: Improving nurses' safe practice in intensive care units. Journal of Caring Sciences, 2(3), 177-185. http://dx.doi.org/10.5681/jcs.2013.022

Mardis, M., Davis, J., Benningfield, B., Elliott, C., Youngstrom, M., Nelson, B., Justice, E. M., \& Riesenberg, L. A. (2015). Shift-to-Shift Handoff Effects on Patient Safety and Outcomes: A Systematic Review. American Journal of Medical Quality, 30. [Epub ahead of print]. http://dx.doi.org/10.1177/1062860615612923 
Matic, J., Davidson, P. M., \& Salamonson, Y. (2011). Review: bringing patient safety to the forefront through structured computerisation during clinical handover. Journal of Clinical Nursing, 20(1-2), 184-189. http://dx.doi.org/10.1111/j.1365-2702.2010.03242.x

McGillis Hall, L., Pedersen, C., \& Fairley, L. (2010). Losing the moment: Understanding interruptions to nurses' work. The Journal of Nursing Administration, 40(4), 169-176. http://dx.doi.org/10.1097/NNA.0b013e3181d41162

National Transition of Care Coalition. (2008). Transition of care measures. [Online] Available: http://www.ntocc.org/Portals/0/PDF/Resources/TransitionsOfCare_Measures.pdf (September 8, 2015)

Ong, M. S., \& Coiera, E. (2011). A systematic review of failures in handoff communication during intra hospital transfers. Joint Commission Journal on Quality and Patient Safety, 37(6), 274-284.

Pothier, D., Monteiro, P., Mooktiar, M., \& Shaw, A. (2005). Pilot study to show the loss of important data in nursing handover. British Journal of Nursing, 14(20), 1090-1093. http://dx.doi.org/10.12968/bjon.2005.14.20.20053

Quattrin, R., Calligaris, L., Londero, C., Zalateu E., \& Brusaferro S. (2014). Shift-to-shift handoff: A comparison between two methods of conveying essential information in a University Hospital in North-eastern Italy. Journal of Hospital Administration, 3(5), 39-46. http://dx.doi.org/10.5430/jha.v3n5p39

Rabøl, L. I., Andersen, M. L., Østergaard, D., Bjørn, B., Lilja, B., \& Mogensen, T. (2011). Descriptions of verbal communication errors between staff. An analysis of 84 root cause analysis-reports from Danish hospitals. BMJ Quality and Safety, 20(3), 268-274. http://dx.doi.org/10.1136/bmjqs.2010.040238

Salzwedel, C., Bartz, H. J., Kühnelt, I., Appel, D., Haupt, O., Maisch, S., \& Schmidt, G. N. (2013). The effect of a checklist on the quality of post-anaesthesia patient handover: A randomized controlled trail. International Journal for Quality in Health Care, 25(2), 176-181. http://dx.doi.org/10.1093/intqhc/mzt009

Siedlecki. S. L., \& Hixson, E. D. (2015). Relationships between Nurses and Physicians Matter. Online Journal of Issues in Nursing, 20(2), 6. http://dx.doi.org/10.3912/OJIN.Vol20No03PPT03

Spooner, A. J., Corley, A., Chaboyer, W., Hammond, N. E., \& Fraser, J. F. (2015). Measurement of the frequency and source of interruptions occurring during bedside nursing handover in the intensive care unit: An observational study. Australian Critical Care, 28(1), 19-23. http://dx.doi.org/10.1016/j.aucc.2014.04.002

Thomas, M. J., Schultz, T. J., Hannaford, N., \& Runciman, W. B. (2013). Failures in transition: learning from incidents relating to clinical handover in acute care. Journal for Healthcare Quality, 35(3), 49-56. http://dx.doi.org/10.1111/j.1945-1474.2011.00189.x 
Westbrook, J. I., Coiera, E., Dunsmuir, W. T., Brown, B. M., Kelk, N., Paoloni, R., \& Tran, C. (2010). The impact of interruptions on clinical task completion. Quality \& Safety in Health Care, 19(4), 284-289. http://dx.doi.org/10.1136/qshc.2009.039255

WHO Collaborating Centre for Patient Safety Solutions. (2007). Communication during patient handovers. [Online]. Available: http://www.who.int/patientsafety/solutions/patientsafety/PS-Solution3.pdf (May 12, 2016)

Wong, M. C., Yee, K. C., \& Turner, P. (2008). Clinical Handover Literature Review, eHealth Services Research Group, University of Tasmania Australia. [Online]. Available: http://www.safetyandquality.gov.au/wp-content/uploads/2008/01/Clinical-Handover-Literatu re-Review-for-release.pdf (March 26, 2016)

Wong, M.C., Turner, P., \& Yee, K.C. (2008). Involving clinicians in the development of an electronic clinical handover system-thinking systems not just technology. Studies in Health Technology and Informatics, 490-495. http://dx.doi.org/10.3233/978-1-58603-864-9-490

Table 1. Distribution of used shift-to-shift methods and actually setting to transmit patient information among respondent physicians (multiple choice)

\begin{tabular}{|c|c|c|}
\hline Shift-to-shift methods actually used & $\mathbf{N}^{\circ}$ & $\mathbf{\%}$ \\
\hline Verbal communication & $71 / 157$ & 45.2 \\
\hline Written note & $41 / 157$ & 26.1 \\
\hline Electronic support & $34 / 157$ & 21.7 \\
\hline Standardized checklist & $4 / 157$ & 2.5 \\
\hline Instant messaging & $4 / 157$ & 2.5 \\
\hline None & $3 / 157$ & 1.9 \\
\hline Handover setting & $\mathbf{N}^{\circ}$ & $\mathbf{\%}$ \\
\hline When my colleague takes over on duty & $64 / 157$ & 40.0 \\
\hline When I finish my ward shift & $54 / 157$ & 33.8 \\
\hline During informal breaks & $14 / 157$ & 8.8 \\
\hline At the end of the round ward & $12 / 157$ & 7.5 \\
\hline Morning report/ward meeting & $5 / 157$ & 3.1 \\
\hline Never & $3 / 157$ & 1.9 \\
\hline Other & $8 / 157$ & 5.0 \\
\hline
\end{tabular}




\section{Macrothink}

Journal of Safety Studies

ISSN 2377-3219

2017, Vol. 3, No. 1

Table 2. Reasons of physicians' experienced obstacles to an effective handover (multiple choice) and some single reason

\begin{tabular}{|l|c|c|}
\hline Obstacles to an effective handover & $\mathbf{N}^{\circ}$ & $\mathbf{2 9}$ \\
\hline Frequent interruptions and distractions & $27 / 107$ & 27.1 \\
\hline Lack of structured policies and procedures about handover & $27 / 107$ & 25.2 \\
\hline Colleague's inability to provide further information when required & $19 / 107$ & 17.7 \\
\hline Handover takes time to other activities & $9 / 107$ & 8.4 \\
\hline “My colleague is not easily accessible when I end my shift” & $6 / 107$ & 5.6 \\
\hline “I finish the shift soon after surgery” & $3 / 107$ & 2.8 \\
\hline Single reasons & & \\
\hline Interpersonal communication problems & \\
\hline Transmission not of objective data but of their interpretation & \\
\hline Fatigue at the end of the shift & \\
\hline Lack of written tools for handover & \\
\hline Lack of electronic support for handover & \\
\hline Shift to shift handover are sometimes quick due to the turnover of surgery or other tasks \\
\hline
\end{tabular}

\section{Copyright Disclaimer}

Copyright for this article is retained by the author(s), with first publication rights granted to the journal.

This is an open-access article distributed under the terms and conditions of the Creative Commons Attribution license (http://creativecommons.org/licenses/by/3.0/). 